\title{
Comparison of Test Your Memory and Montreal Cognitive Assessment Measures in Parkinson's Disease
}

\author{
Emily J. Henderson, ${ }^{1}$ Howard Chu, ${ }^{2}$ Daisy M. Gaunt, ${ }^{3}$ Alan L. Whone, ${ }^{4}$ \\ Yoav Ben-Shlomo, ${ }^{1}$ and Veronica Lyell ${ }^{5}$ \\ ${ }^{1}$ School of Social and Community Medicine, University of Bristol, Canynge Hall, 39 Whatley Road, Bristol BS8 2PS, UK \\ ${ }^{2}$ Taunton and Somerset NHS Foundation Trust, Musgrove Park Hospital, Parkfield Drive, Taunton, Somerset TA1 5DA, UK \\ ${ }^{3}$ Bristol Randomised Trials Collaboration, School of Social and Community Medicine, Canynge Hall, 39 Whatley Road, \\ Bristol BS8 2PS, UK \\ ${ }^{4}$ Movement Disorders Group, Bristol Brain Centre, Southmead Hospital, Bristol BS10 5NB, UK \\ ${ }^{5}$ Royal United Hospital NHS Foundation Trust, Combe Park, Bath BA1 3NG, UK
}

Correspondence should be addressed to Emily J. Henderson; emily.henderson@bristol.ac.uk

Received 1 February 2016; Accepted 24 May 2016

Academic Editor: Marjan Jahanshahi

\begin{abstract}
Copyright (C) 2016 Emily J. Henderson et al. This is an open access article distributed under the Creative Commons Attribution License, which permits unrestricted use, distribution, and reproduction in any medium, provided the original work is properly cited.

Background. MoCA is widely used in Parkinson's disease (PD) to assess cognition. The Test Your Memory (TYM) test is a cognitive screening tool that is self-administered. Objectives. We sought to determine (a) the optimal value of TYM to discriminate between PD patients with and without cognitive deficits on MoCA testing, (b) equivalent MoCA and TYM scores, and (c) interrater reliability in TYM testing. Methods. We assessed the discriminant ability of TYM and the equivalence between TYM and MoCA scores and measured the interrater reliability between three raters. Results. Of the 135 subjects that completed both tests, $55 \%$ had cognitive impairment according to MoCA. A MoCA score of 25 was equivalent to a TYM score of 43-44. The area under the receiver operator characteristic (ROC) curve for TYM to differentiate between PD-normal and PD-cognitive impairment was 0.82 (95\% CI 0.75 to 0.89). The optimal cutoff to distinguish PD-cognitive impairment from PD-normal was $\leq 45$ (sensitivity $90.5 \%$, specificity $59 \%$ ) thereby correctly classifying $76.3 \%$ of patients with PD-cognitive impairment. Interrater agreement was high (0.97) and TYM was completed in under 7 minutes (interquartile range 5.33 to 8.52 minutes). Conclusions. The TYM test is a useful and less resource intensive screening test for cognitive deficits in PD.
\end{abstract}

\section{Introduction}

Cognitive impairment in Parkinson's disease (PD) is common and associated with functional impairment and poor quality of life $[1,2]$. The spectrum of dysfunction ranges from executive dysfunction to mild cognitive impairment (MCI) seen even in early PD (PD-MCI), through to Parkinson's disease dementia (PDD). PDD has a cumulative incidence of $80 \%$ and is associated with significant morbidity, mortality, and carer stress [1,3-5]. As the presence of $\mathrm{MCI}$ is associated with the development of dementia [6-8] and cognitive deficits impact quality of life [9], accurate identification of those with early cognitive changes is important to facilitate early planning, support, and intervention.
The Montreal Cognitive Test (MoCA) is increasingly used to screen for cognitive deficits, largely replacing the less sensitive Mini Mental State Examination (MMSE) [10-12]. MoCA takes 10-15 minutes to administer and assesses seven cognitive domains: visuospatial/executive (5 points), naming ( 3 points), attention (6 points), language ( 3 points), abstraction ( 2 points), memory ( 5 points), and orientation ( 6 points), yielding a total possible score of 30 . One point is added if the individual has $\leq 12$ years of education. Two studies have examined MoCA as a screening test for cognitive impairment in PD. To identify possible PD-MCI with $>80 \%$ sensitivity, MoCA cutoff scores of 26/27 [10] or $<26 / 30$ [11] have been advocated. The Movement Disorder Society (MDS) Task Force for the diagnosis of PD-MCI (level 1 criteria) supports 
the use of MoCA to demonstrate global cognitive deficits in a clinical setting [13].

The Test Your Memory (TYM) (available from http:// www.tymtest.com/) scale is a self-administered test that is validated in Alzheimer's disease (AD) and has been used in different regions (including in other languages) and clinical settings [14-16]. TYM's distinct advantage is that it reduces demands on clinical time as it can be supervised by nonclinical staff. TYM tests the same domains as MoCA: orientation (10 points), ability to copy a sentence (2 points), semantic knowledge (3 points), calculation ( 4 points), verbal fluency ( 4 points), similarities ( 4 points), naming (5 points), visuospatial abilities ( 2 tasks, total 7 points), and recall of a copied sentence ( 6 points). Ability to complete the test without assistance is scored (executive function, 5 points), yielding a total possible score of 50 . For both tests, a higher score indicates better performance. With constraints on clinical time, TYM may represent a helpful additional or alternative tool to screen for cognitive deficits in PD.

This substudy sought to determine the ability of TYM to detect cognitive deficits in $\mathrm{PD}$, determine equivalence between TYM and MoCA scores in PD, and assess interrater reliability of TYM scoring.

\section{Materials and Methods}

2.1. Study Population. We undertook a diagnostic test study nested within the ReSPonD trial, a double blind randomised controlled trial of Rivastigmine versus placebo to stabilise gait in people with PD [17]. Patients were invited to attend a screening clinic appointment if they appeared to meet the eligibility criteria for the ReSPonD trial. We sought to identify participants with idiopathic Parkinson's who did not have established dementia, were not treated with cholinesterase inhibitors, were able to walk $18 \mathrm{~m}$, and had been stable on PD medication for 2 weeks. Patients were excluded if they had neurological, visual, or orthopaedic problems that interfered with balance or gait or were non-English speaking (cognitive tests were performed in English). Potential participants were identified from community and hospital settings, through registers and publicity campaigns.

Interested participants were sent an information pack and, if interested, had their eligibility checked by telephone. They were then invited for a face-to-face assessment when they completed the MoCA as part of the screening protocol. All patients at this visit were invited to participate in the TYM study regardless of their subsequent involvement in the ReSPonD trial. We excluded patients from the drug trial who had overt PD-dementia, the diagnosis of which was operationalised using the Movement Disorder Society Task Force definition of decreased cognition of sufficient severity to impair daily life [18]. Patients with a low MoCA score without clinically overt dementia (on global clinical assessment) were not excluded. Ethical approval was granted from the South West Central Bristol Research Ethics Committee and written informed consent was obtained from participants.

2.2. Procedures. Basic demographic information was obtained for all participants who were assessed in a clinically defined "on" medication state. More in-depth demographic and clinical information was gathered for the participants who subsequently enrolled in the RCT. MoCA and TYM were performed by trained research staff in a variable but nonrandomised order. The MoCA was completed by a registrar in geriatric medicine or trained research nurse, both of whom supervised and timed the TYM tests. All TYM tests were scored by a medical student (HC). To assess interrater reliability, 30\% ( $n=40 / 135)$ were additionally scored by two other individuals, a consultant geriatrician (VL) and a research assistant with no clinical experience, both of whom were provided with the published marking instructions only.

2.3. Statistical Analysis. Baseline data are described as mean \pm SD if normally distributed or as median interquartile range (25th percentile, 75th percentile) if skewed. As TYM and MoCA are scored on different scales, equipercentile equating with log-linear smoothing [19] was undertaken using the "equate" package developed for " $R$ ". Equally ranked percentiles are considered equivalent for the two scores and a conversion table is produced.

We used published screening criteria to classify participants as "PD-normal" (MoCA score 26-30), "PD-MCI" (MoCA score 21-25), and "PDD" (MoCA < 21) [11]. We then grouped PD-MCI and PDD into one group ("PDcognitive impairment"). A receiver operating characteristic (ROC) was plotted and the area under the curve (AUC) was calculated to determine the ability of worsening TYM score to discriminate between PD-normal (MoCA score 26-30) versus $\mathrm{PD}$-cognitive impairment (MoCA score $\leq 25)$. The optimal TYM screening cutoff was calculated by maximising Youden's $J$ statistic [20] which gives equal weighting to sensitivity and specificity.

To assess reliability of TYM, we calculated the intraclass correlation coefficient (ICC) for interrater agreement using a two-way random-effects model assuming that the raters were randomly drawn from the population. The ICC is the ratio of intersubject variability to the total variability, defined as the sum of the intersubject variability, the between rater variability, and error variability. An ICC greater than 0.80 is regarded as indicative of high reliability [21]. Absolute difference between raters on TYM was calculated using a gold standard rater (VL) and subtracting the individual scores of the other two raters (i.e., VL TYM score minus other rater TYM scores). Statistical analysis was performed using Stata version 13.1 and " $R$ ” [22].

\section{Results}

3.1. Screening and Demographic Characteristics. Overall, 931 patients were screened for potential inclusion in the study. Of these, 500 (54\%) did not meet the trial eligibility criteria, regardless of whether they wished to participate. Of the remaining 301 who were not enrolled and were potentially eligible, 143 did not reply to the initial invitation to attend and 158 declined to participate. Therefore, 135 attended for faceto-face screening, of whom 130 went on to participate in the ReSPonD trial. Of the 5 who did not subsequently enroll in the drug trial, $n=1$ declined, $n=2$ had likely PDD, and 


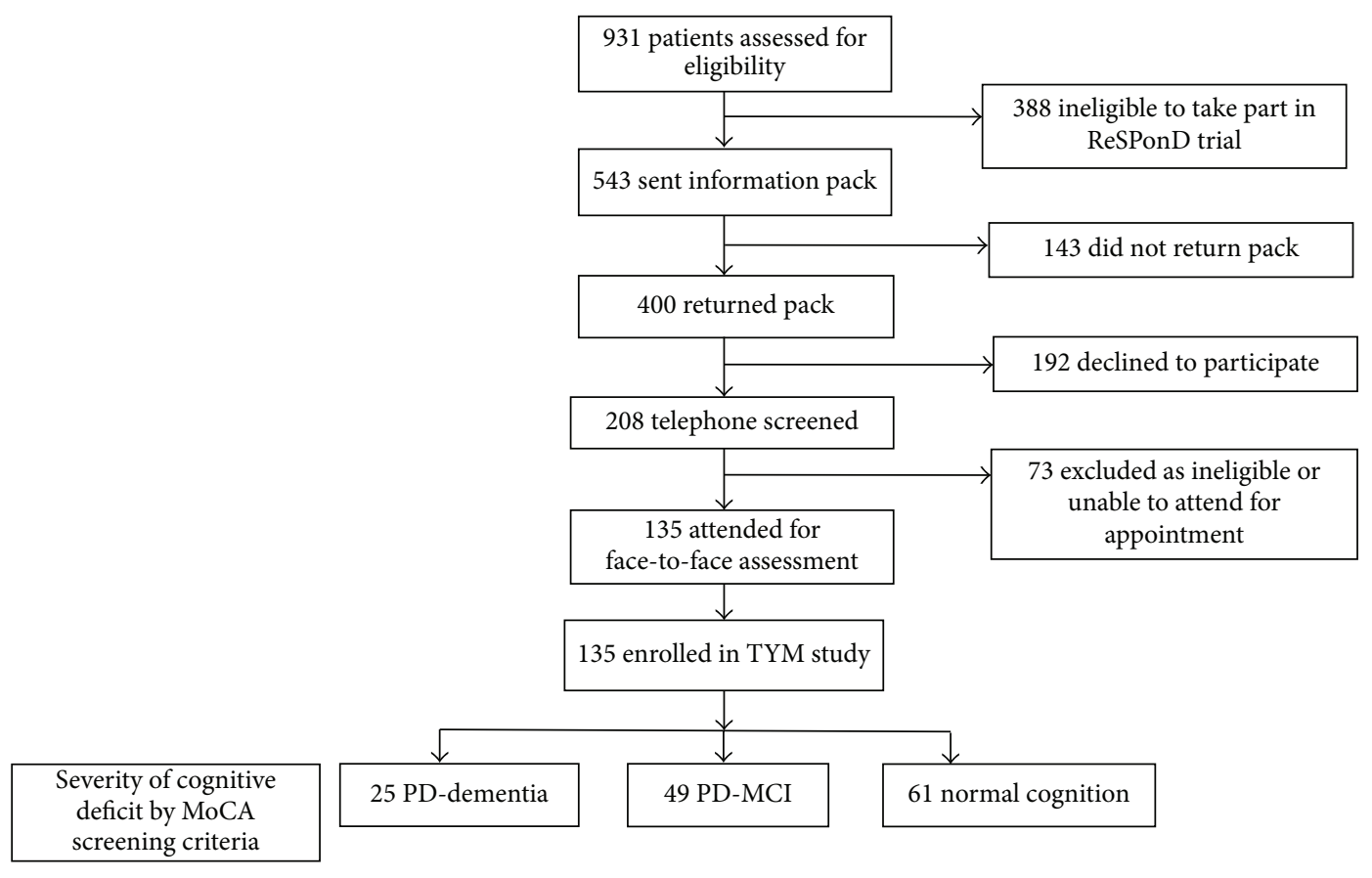

Figure 1: Patient flow.

TABLE 1: Baseline demographic data.

\begin{tabular}{lc}
\hline & Participants $(n=135)$ \\
\hline Mean age & $70.0(8.1)$ \\
Sex $(n$ female (\%)) & $51(38 \%)$ \\
Caucasian ethnicity & $134(99 \%)$ \\
Age at leaving school & $16(15-17)$ \\
Montreal cognitive assessment (total score) & $25(22-27)$ \\
"PD-normal impairment" (MoCA 26-30) & $61(45 \%)$ \\
"PD-cognitive impairment" (MoCA $\leq 25)$ & $74(55 \%)$ \\
Test your memory (total score) & $43(39-46)$ \\
Total MDS-UPDRS (total score) & $90(74-106)^{*}$ \\
Duration of PD (yrs) & $9(5-13)^{*}$ \\
\hline
\end{tabular}

${ }^{*} n=130$

2 were unable to walk $18 \mathrm{~m}$ without an aid. All 5 however participated in the TYM study. Participant recruitment is shown in Figure 1.

The characteristics of our cohort are summarised in Table 1. Participants were predominantly Caucasian with a mean (SD) age of 70 (8.1) years. The median MoCA score was 25 and median TYM score was 43.

3.2. Test Distributions. MoCA and TYM assessments were performed on all 135 participants. MoCA scores ranged from 7 to 30 and TYM ranged from 15 to 50 . Both measures were negatively skewed. Using the published screening cutoffs for MoCA [11], $n=25$ (19\%) had deficits consistent with PDD, $n=49(36 \%)$ had deficits consistent with MCI, and $n=$ $61(45 \%)$ had normal cognition. The median time taken to complete TYM was 6.53 mins (interquartile range 5.33 to

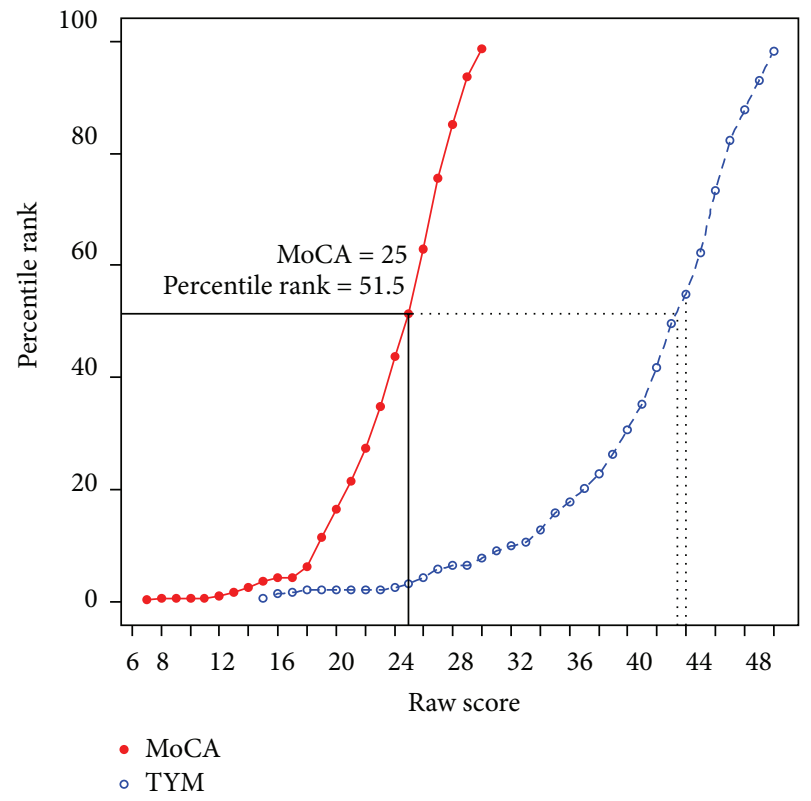

FIGURE 2: Corresponding raw scores and percentile rank for TYM and MoCA.

8.52 mins). $47 \%$ ( $n=63$ ) of patients required some degree of assistance to complete the test.

3.3. Translation between MoCA and TYM. Corresponding MoCA and TYM scores, after log-linear smoothed equipercentile equating, are shown in Figure 2 and Table 2. Extrapolated data are shown in italics in Table 2 corresponding to a TYM score of $<15$. A MoCA score of 25 (the upper limit for 
TABLE 2: Equivalent TYM and MoCA scores.

\begin{tabular}{|c|c|c|c|}
\hline TYM & MoCA & TYM & MoCA \\
\hline 0 & 0 & 25 & 15 \\
\hline 1 & 1 & 26 & 16 \\
\hline 2 & 2 & 27 & 16 \\
\hline 3 & 3 & 28 & 17 \\
\hline 4 & 4 & 29 & 17 \\
\hline 5 & 5 & 30 & 18 \\
\hline 6 & 6 & 31 & 18 \\
\hline 7 & 7 & 32 & 19 \\
\hline 8 & 7 & 33 & 19 \\
\hline 9 & 8 & 34 & 20 \\
\hline 10 & 8 & 35 & 20 \\
\hline 11 & 9 & 36 & 21 \\
\hline 12 & 9 & 37 & 21 \\
\hline 13 & 10 & 38 & 22 \\
\hline 14 & 10 & 39 & 22 \\
\hline 15 & 11 & 40 & 23 \\
\hline 16 & 11 & 41 & 23 \\
\hline 17 & 12 & 42 & 24 \\
\hline 18 & 12 & 43 & 25 \\
\hline 19 & 12 & 44 & 25 \\
\hline 20 & 13 & 45 & 26 \\
\hline 21 & 13 & 46 & 27 \\
\hline 22 & 14 & 47 & 27 \\
\hline 23 & 14 & 48 & 28 \\
\hline 24 & 15 & 49 & 29 \\
\hline 25 & 15 & 50 & 30 \\
\hline
\end{tabular}

Italics indicate extrapolated data.

screening PD-MCI) corresponds to a TYM score of 43-44, highlighted in bold.

3.4. Sensitivity and Specificity of TYM. The area under the ROC curve (Figure 3) for TYM to differentiate between PDnormal impairment and PD-cognitive impairment as defined by MoCA was 0.82 (95\% CI 0.75 to 0.89 ). The maximised Youden's $J$ statistic with sensitivity $90.5 \%$ and specificity $59.0 \%$ giving optimum accuracy was a TYM score of 45 , which correctly classified $76.3 \%$ of patients with PD-cognitive impairment.

3.5. Interrater Reliability. The ICC for absolute agreement (ICC $=0.97,95 \%$ CI 0.94 to $0.99, p<0.001$ ) was high, indicating excellent scoring reliability. The median (IQR) difference between the gold standard rater (VL) and the other raters was -1 ( -2 to 0$)$ in both cases.

\section{Conclusions}

MoCA is established and advocated as a screening test for cognitive deficits in PD. The main advantage of the TYM test over MoCA and other screening tests is that it is selfadministered, being supervised by nonclinical staff, and

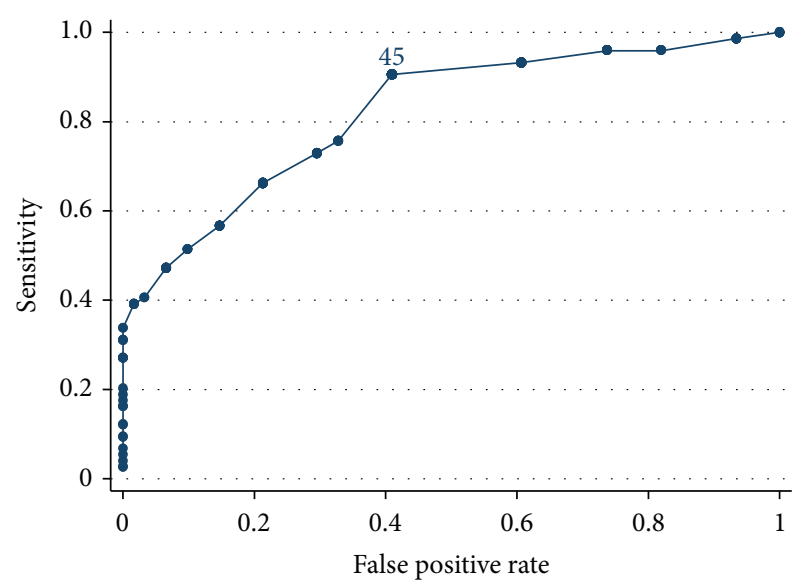

FIGURE 3: ROC curve showing the sensitivity and specificity of different TYM scores for PD-normal (MoCA 26-30) or PD-cognitive impairment $(\mathrm{MoCA} \leq 25)$. Labelled data point $(\mathrm{TYM}=45)$ gives optimum sensitivity and specificity.

can be completed whilst waiting at clinic before seeing a specialist. We have established equivalent scores for TYM with MoCA and assessed the discriminant ability of TYM to detect cognitive deficits in PD. Our results suggest that a TYM score of $\leq 45$ identifies MCI level cognitive deficits with a sensitivity of $90.5 \%$ and specificity of $59.0 \%$. The relatively low specificity is appropriate for the TYM test's role as a screening test. Using TYM can avoid the need for further testing in many patients; those below the cutoff can be assessed further with MoCA or other tools. If motor problems such as severe tremor affect completion of the writing and drawing tasks, TYM can be completed by another individual under direction from the patient. Neither TYM nor MoCA showed notable floor or ceiling effects in this population. The time taken to complete the test was acceptable [12] and comparable to previously published data [16] even in this PD population. This is the first study that we are aware of that has examined the utility of TYM in PD. In contrast to a previous study [10], a substantial proportion of this cohort (55\%) screened positive for cognitive deficits using the MoCA. Despite excluding those with known PDD, our participants had a broad range of cognitive dysfunction severity, which enhances the generalisability of the results.

This study has several limitations. We excluded people with previously diagnosed PDD as they were not eligible to take part in the drug trial in which this substudy was nested. With fewer people with very pronounced cognitive deficits, equivalent scores in the lower range should be interpreted cautiously and this may influence the generalisability of the results. However, we feel that our population of PD patients without dementia but with falls (which are associated with cognitive impairment) represents the group in whom screening for deficits is of most clinical value. We have not compared the TYM to a "gold-standard" test for PD-MCI [23] and PDD [18], but rather to another screening test (albeit one recommended in the diagnostic criteria [level 1] set out by the MDS) [12]. Published MoCA cutoff values for PD-MCI vary slightly between studies. We used a MoCA cutoff score 
of 26 and may therefore have slightly overestimated those with cognitive impairment. We did not measure the time taken to complete MoCA as a comparison. Although TYM completion took less than 7 minutes, it is probable that people with more severe cognitive deficits would have taken longer.

We would still recommend using the MoCA if concerns are raised regarding cognition as this is the recommended standard validated cognitive screening test in PD [12], which stands alone as a minimum assessment, takes $<15$ minutes to complete, measures major cognitive domains, and can identify subtle cognitive impairment. Observation of the completion of a cognitive test may afford a clinician further insight into the cognitive changes. Our results suggest that the TYM also meets these criteria, may be faster, and, as it does not require specialist supervision, could further support detection of cognitive deficits in PD. Accurate identification of individuals who require further cognitive assessment is a necessary component of both research testing and clinical testing. Where clinical resource limitations preclude the use of the MoCA, use of the TYM test in PD may be a valuable tool.

\section{Competing Interests}

The ReSponD trial in which this study was nested was funded by a Parkinson's UK career development award (F1003) awarded to Emily J. Henderson. Other grants have been received from North Bristol NHS Trust and The British Geriatrics Society. Emily J. Henderson is an employee at the Royal United Hospital NHS Foundation Trust, Combe Park, Bath BA1 3NG, UK, and the University of Bristol. She was on the advisory board of Profile Pharma. Howard Chu works for Taunton and Somerset NHS Foundation Trust, Musgrove Park Hospital, Parkfield Drive, Taunton, Somerset TA1 5DA, UK. Veronica Lyell is an employee at the Royal United Hospital NHS Foundation Trust, Combe Park, Bath BA1 3NG, UK. Daisy M. Gaunt works at Bristol Randomised Trials Collaboration, School of Social and Community Medicine, Canynge Hall, 39 Whatley Road, Bristol BS8 2PS, UK. Alan L. Whone works for the North Bristol NHS Trust, Bristol BS10 5NB, UK, received grants from Parkinson's UK, and is on the advisory boards of Pfizer and Medtronic. He received honoraria from Lundbeck. Yoav Ben-Shlomo is a Member of the Department of Health MS Risk Sharing Scheme and also a Member of the National Joint Register scientific advisory board. He works at the University of Bristol, and received royalties from book publications from Oxford University Press and Wiley and Co. He received multiple grants from National Institute of Health Research, MRC, Parkinson's UK, Brain Tumour Charity, US National Institutes of Health (National Institute on Aging), and UK Economic and Social Research Council.

\section{Authors' Contributions}

Emily J. Henderson was responsible for conception, organization, and execution of the research project; design and execution of statistical analysis; and writing of the first draft, review, and critique of the paper. Howard Chu was responsible for execution of the research project. Daisy M. Gaunt carried out the design, execution, review, and critique of the statistical analysis and review and critique of the paper. Alan L. Whone was responsible for organization and execution of the research project and review and critique of the paper. Yoav Ben-Shlomo was responsible for organization and execution of the research project and review and critique of the statistical analysis and of the paper. Veronica Lyell was responsible for conception, organization, and execution of the research project; design, review, and critique of the statictical analysis; and review and critique of the paper.

\section{Acknowledgments}

The authors would like to thank Michael Lawton and Celia Gregson for their valuable input and Parkinson's UK for their funding support. This study was nested within a trial funded by Parkinson's UK.

\section{References}

[1] M. A. Hely, W. G. J. Reid, M. A. Adena, G. M. Halliday, and J. G. L. Morris, "The Sydney multicenter study of Parkinson's disease: the inevitability of dementia at 20 years," Movement Disorders, vol. 23, no. 6, pp. 837-844, 2008.

[2] E. Rosenthal, L. Brennan, S. Xie et al., "Association between cognition and function in patients with Parkinson disease with and without dementia," Movement Disorders, vol. 25, no. 9, pp. 1170-1176, 2010.

[3] L. P. Oosterveld, J. C. Allen, G. Reinoso et al., "Prognostic factors for early mortality in Parkinson's disease," Parkinsonism and Related Disorders, vol. 21, no. 3, pp. 226-230, 2015.

[4] J. Gratwicke, M. Jahanshahi, and T. Foltynie, "Parkinson's disease dementia: a neural networks perspective," Brain, vol. 138, pp. 1454-1476, 2015.

[5] D. Aarsland, K. Brønnick, U. Ehrt et al., "Neuropsychiatric symptoms in patients with Parkinson's disease and dementia: frequency, profile and associated care giver stress," Journal of Neurology, Neurosurgery and Psychiatry, vol. 78, no. 1, pp. 3642, 2007.

[6] C. C. Janvin, J. P. Larsen, D. Aarsland, and K. Hugdahl, "Subtypes of mild cognitive impairment in Parkinson's disease: progression to dementia," Movement Disorders, vol. 21, no. 9, pp. 1343-1349, 2006.

[7] D. Aarsland, K. Brønnick, J. P. Larsen, O. B. Tysnes, and G. Alves, "Cognitive impairment in incident, untreated parkinson disease: the Norwegian parkwest study," Neurology, vol. 72, no. 13, pp. 1121-1126, 2009.

[8] C. H. Williams-Gray, J. R. Evans, A. Goris et al., "The distinct cognitive syndromes of Parkinson's disease: 5 year follow-up of the CamPaIGN cohort," Brain, vol. 132, no. 11, pp. 2958-2969, 2009.

[9] A. Schrag, M. Jahanshahi, and N. Quinn, "What contributes to quality of life in patients with Parkinson's disease?" Journal of Neurology, Neurosurgery \& Psychiatry, vol. 69, no. 3, pp. 308312, 2000.

[10] S. Hoops, S. Nazem, A. D. Siderowf et al., "Validity of the MoCA and MMSE in the detection of MCI and dementia in Parkinson disease," Neurology, vol. 73, no. 21, pp. 1738-1745, 2009.

[11] J. C. Dalrymple-Alford, M. R. MacAskill, C. T. Nakas et al., "The MoCA: well-suited screen for cognitive impairment in 
Parkinson disease," Neurology, vol. 75, no. 19, pp. 1717-1725, 2010.

[12] K. L. Chou, M. M. Amick, J. Brandt et al., "A recommended scale for cognitive screening in clinical trials of Parkinson's disease," Movement Disorders, vol. 25, no. 15, pp. 2501-2507, 2010.

[13] I. Litvan, J. G. Goldman, A. I. Tröster et al., "Diagnostic criteria for mild cognitive impairment in Parkinson's disease: Movement Disorder Society Task Force guidelines," Movement Disorders, vol. 27, no. 3, pp. 349-356, 2012.

[14] H. Hanyu, M. Maezono, H. Sakurai, K. Kume, H. Kanetaka, and T. Iwamoto, "Japanese version of the Test Your Memory as a screening test in a Japanese memory clinic," Psychiatry Research, vol. 190, no. 1, pp. 145-148, 2011.

[15] G. van Schalkwyk, H. Botha, and S. Seedat, "Comparison of 2 dementia screeners, the test your memory test and the minimental state examination, in a primary care setting," Journal of Geriatric Psychiatry and Neurology, vol. 25, no. 2, pp. 85-88, 2012.

[16] J. Brown, G. Pengas, K. Dawson, L. A. Brown, and P. Clatworthy, "Self administered cognitive screening test (TYM) for detection of Alzheimer's disease: cross sectional study," The British Medical Journal, vol. 338, no. 7708, Article ID b2030, 2009.

[17] E. J. Henderson, S. R. Lord, J. C. T. Close, A. D. Lawrence, A. Whone, and Y. Ben-Shlomo, "The ReSPonD trial - rivastigmine to stabilise gait in Parkinson's disease a phase II, randomised, double blind, placebo controlled trial to evaluate the effect of rivastigmine on gait in patients with Parkinson's disease who have fallen," BMC Neurology, vol. 13, article 188, 2013.

[18] B. Dubois, D. Burn, C. Goetz et al., "Diagnostic procedures for Parkinson's disease dementia: recommendations from the Movement Disorder Society Task Force," Movement Disorders, vol. 22, no. 16, pp. 2314-2324, 2007.

[19] M. J. Kolen and R. L. Brennan, Test Equating, Scaling, and Linking: Methods and Practices, Springer, New York, NY, USA, 2014.

[20] W. J. Youden, "Index for rating diagnostic tests," Cancer, vol. 3, no. 1, pp. 32-35, 1950.

[21] H.-Y. Kim, "Statistical notes for clinical researchers: evaluation of measurement error 1: using intraclass correlation coefficients," Restorative Dentistry \& Endodontics, vol. 38, no. 2, pp. 98-102, 2013.

[22] RStudio, Version 0.99.473- (C) 2009-2015 RStudio, Inc., (n.d.).

[23] I. Litvan, D. Aarsland, C. H. Adler et al., "MDS task force on mild cognitive impairment in Parkinson's disease: critical review of PD-MCI," Movement Disorders, vol. 26, no. 10, pp. 1814-1824, 2011. 


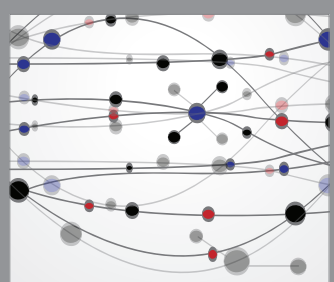

The Scientific World Journal
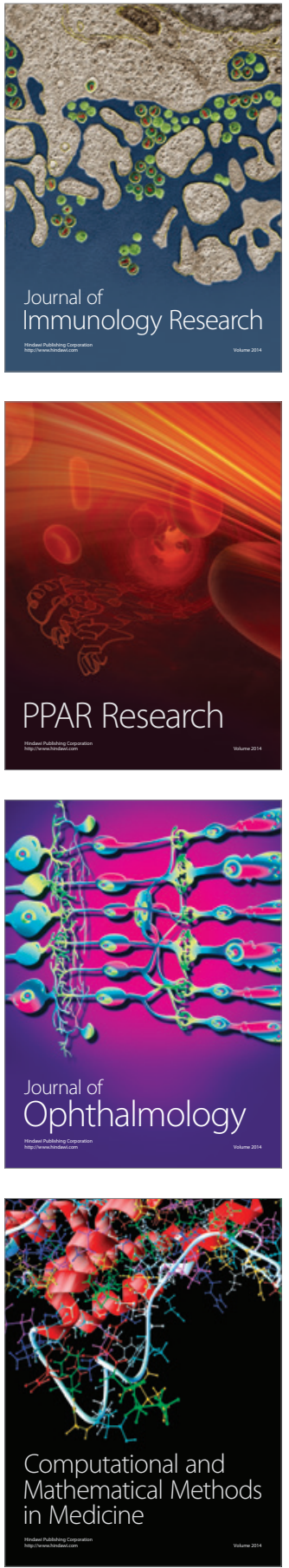

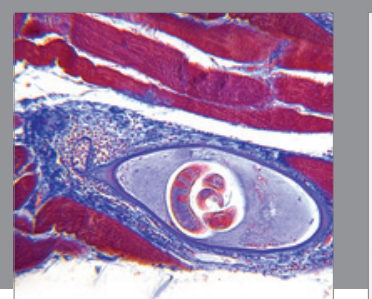

Gastroenterology Research and Practice

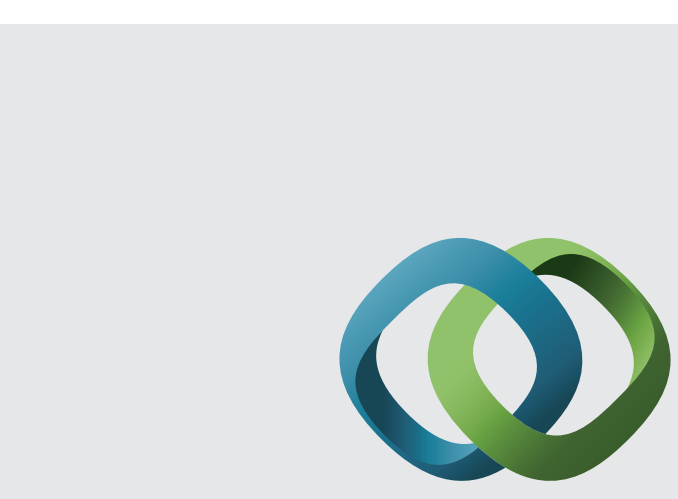

\section{Hindawi}

Submit your manuscripts at

http://www.hindawi.com
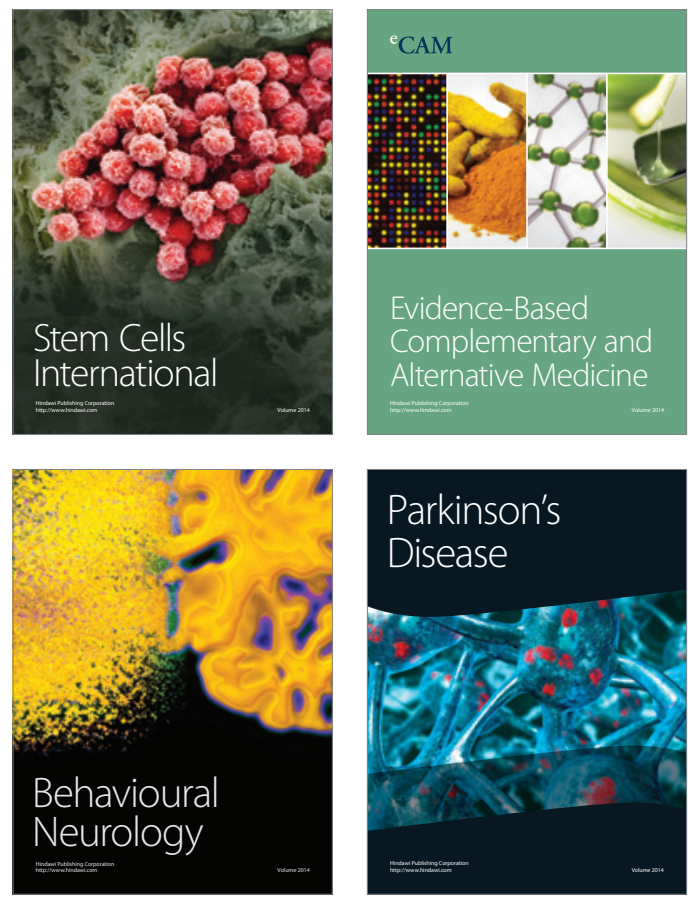
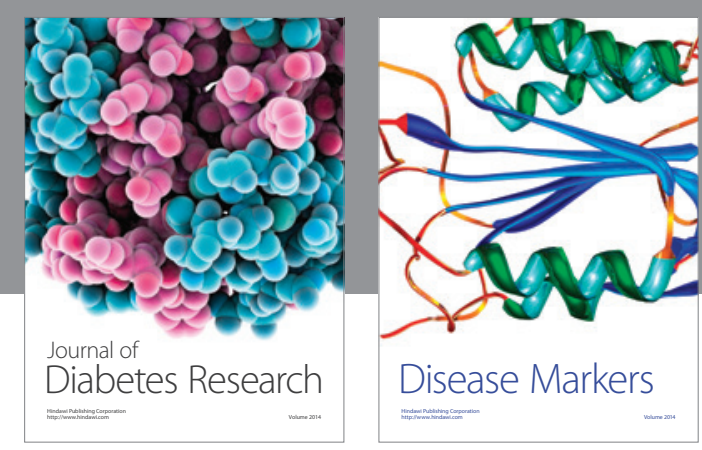

Disease Markers
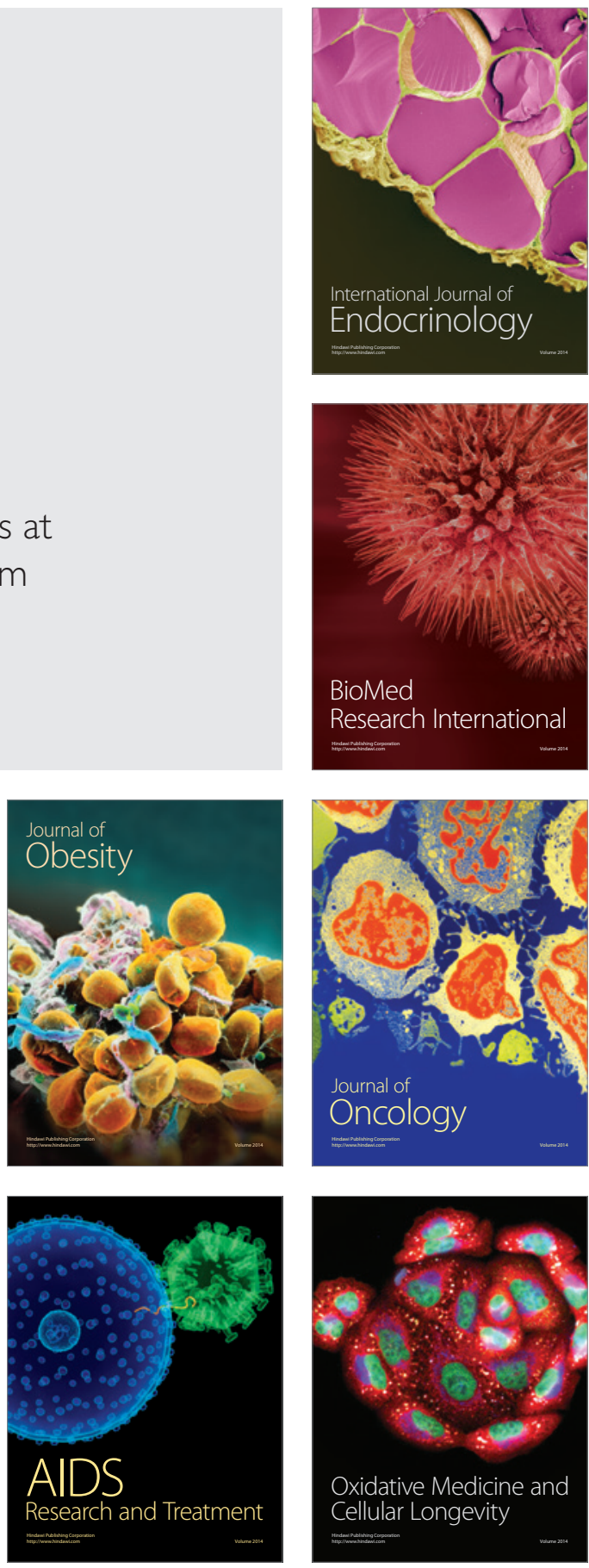